\title{
RADIAL VELOCITIES AND PULSATION EPHEMERIDES OF 11 FIELD RR Lyrae STARS
}

\author{
Bi-Qing For ${ }^{1}$, George W. Preston ${ }^{2}$, And Christopher Sneden ${ }^{1}$ \\ ${ }^{1}$ Department of Astronomy, University of Texas, Austin, TX 78712, USA; biqing@astro.as.utexas.edu \\ ${ }^{2}$ Carnegie Observatories, 813 Santa Barbara Street, Pasadena, CA 91101, USA \\ Received 2011 March 4; accepted 2011 April 4; published 2011 May 27
}

\begin{abstract}
We present new radial velocities (RVs), improved pulsation periods, and reference epochs of 11 field RR Lyrae ab-type variables: AS Vir, BS Aps, CD Vel, DT Hya, RV Oct, TY Gru, UV Oct, V1645 Sgr, WY Ant, XZ Aps, and Z Mic. This study is based on high-resolution spectra obtained with the echelle spectrograph of the $2.5 \mathrm{~m} \mathrm{du}$ Pont telescope at Las Campanas Observatory. We obtained $\sim 200$ spectra per star (i.e., a total of $\sim 2300$ spectra), distributed more or less uniformly throughout their pulsation cycles. RV curves and photometric light curves phased to our new ephemerides are presented for all program stars. In a subsequent paper, we will use these spectra to derive stellar atmospheric parameters and chemical compositions throughout the pulsational cycles, based purely on spectroscopic constraints.
\end{abstract}

Key words: stars: horizontal-branch - stars: Population II - stars: variables: RR Lyrae - techniques: radial velocities

Online-only material: color figures, machine-readable table

\section{INTRODUCTION}

RR Lyraes (RR Lyr), named after their prototype, are old, lowmass stars that reside in the instability strip of the horizontal branch (HB). They are powerful tools in the studies of many fundamental astrophysical problems. Due to their variability and relatively high luminosity, they are easily identified even at large distances. Their small dispersion in intrinsic mean luminosity makes them good standard candles in contrast to other stellar tracers, such as M giants (Majewski et al. 2003). In addition to the distance scale, RR Lyr play an important role in the study of Galactic structure and formation. They are generally used to trace the spatial and kinematic distribution of the old stellar populations of the Galactic disk and halo components. For example, recent optical RR Lyrae surveys, such as QUEST (Vivas et al. 2004) and SDSS (Ivezić et al. 2004), have revealed halo substructures and dynamically young stellar streams that are associated with the formation of the outer halo.

RR Lyr are also commonly used to study the chemical evolution of the disk and halo of our Milky Way. This effort began with the pioneering low-resolution spectroscopic survey by Preston (1959), who introduced the $\Delta S$ index that describes the relation between hydrogen and calcium $K$-line absorption strengths. The $\Delta S$ index varies during RR Lyr pulsational cycles, so the standard $\Delta S$ index is defined at light minimum (i.e., near phase 0.8 ). High-resolution studies generally have concentrated on limited phases near minimum light, because of the relatively slow variations in photometric effective temperature that occur at these pulsation phases.

Our work in this area began as an investigation of the systematics of chemical abundances along the HB in the Galactic Halo (For \& Sneden 2010). The primary objectives of that paper were to investigate any abundance anomalies in nonvariable RHB and BHB stars, to derive masses of these stars and to determine the red and blue edges of the RR Lyr instability strip. They concluded that (1) the abundance ratios of these stars are generally consistent with those of similar-metallicity field stars in different evolutionary stages, (2) the stars possess masses of $\sim 0.5 M_{\odot}$, and (3) the effective temperatures for the red and blue edges of HB stars in the metallicity range $-2.5 \lesssim$ $[\mathrm{Fe} / \mathrm{H}] \lesssim-0.8$ are $5900 \mathrm{~K}$ and $7400 \mathrm{~K}$, respectively.

We are applying the analytical techniques of For \& Sneden (2010) to a controlled sample of RR Lyr stars. The spectra have been gathered by GWP for his investigation of many issues in RR Lyr atmospheric dynamics, such as shocks, turbulence, and the Blazhko effect. This RR Lyr spectral study was also initiated partly to better understand the nature of a carbon-rich and $s$-process-rich RRab star, TY Gru (Preston et al. 2006b). This star was identified as CS 22881-071 in the HK objective-prism survey (Beers et al. 1992) and was initially included in the study of chemical abundance of a sample metal-poor red HB stars (Preston et al. 2006a). The enrichment of carbon and $n$-capture species suggests that this star might have gone through binary mass transfer from a primary star during its asymptotic giant branch (AGB) evolution (Preston et al. 2006b, and references therein). To further investigate the abundance anomalies as seen in TY Gru and to detect the possible orbital motion caused by the relic companion of an AGB star (Preston 2011), GWP selected a sample of RRab stars with $P \sim 0.57$ days that are broadly representative of the metal-poor halo. Numerous observations at all pulsation phases provide a data set that can be used to investigate the dependence of derived abundances on the various thermodynamic conditions that occur during pulsation cycles.

In this paper, we present radial velocities (RVs) and improved ephemerides of 11 field RRab stars. In a subsequent paper, we will report stellar parameter and chemical abundance analyses throughout the pulsational cycles (B.-Q. For et al. 2011, in preparation). We provide the basic information on targets and describe the observations and reduction methods in Sections 2 and 3. In Section 4, we present the derived RVs and improved ephemerides.

\section{TARGETS AND OBSERVATIONS}

The observations were made with echelle spectrograph of the du Pont $2.5 \mathrm{~m}$ telescope at the Las Campanas Observatory (LCO) during 2006-2009. We used this instrument configured with the $1.5 \times 4^{\prime \prime}$ entrance slit, which gives a resolving power 
Table 1

Program Stars

\begin{tabular}{lccccc}
\hline \hline Star & $\begin{array}{c}\text { R.A. (J2000) } \\
(\mathrm{hr} \mathrm{m} \mathrm{s})\end{array}$ & $\begin{array}{c}\text { Decl. }(\mathrm{J} 2000) \\
\left({ }^{\prime \prime \prime}\right)\end{array}$ & $\begin{array}{c}V_{\max }{ }^{\mathrm{a}} \\
(\mathrm{mag})\end{array}$ & $\begin{array}{c}V_{\mathrm{amp}}{ }^{\mathrm{b}} \\
(\mathrm{mag})\end{array}$ & Note \\
\hline CD Vel & 094438.24 & -455237.2 & 11.66 & 0.87 & Blazhko $^{\mathrm{c}}$ \\
WY Ant & 101604.95 & -294342.4 & 10.37 & 0.85 & $\ldots$ \\
DT Hya & 115400.18 & -311540.0 & 12.53 & 0.98 & $\ldots$ \\
AS Vir & 125245.86 & -101536.4 & 11.66 & 0.72 & Blazhko \\
RV Oct & 134631.75 & -842406.4 & 10.53 & 1.13 & Blazhko \\
XZ Aps & 145205.43 & -794046.6 & 11.94 & 1.1 & $\ldots$ \\
BS Aps & 162051.51 & -714015.8 & 11.9 & 0.68 & Blazhko \\
UV Oct & 163225.53 & -835410.5 & 9.19 & 0.82 & Blazhko \\
V1645 Sgr & 202044.47 & -410705.7 & 10.99 & 0.84 & Blazhko \\
Z Mic & 211622.71 & -301703.1 & 11.32 & 0.64 & Blazhko \\
TY Gru & 221639.42 & -395618.0 & $13.6^{\mathrm{d}}$ & $0.9^{\mathrm{d}}$ & Blazhko \\
\hline
\end{tabular}

Notes.

a Maximum light in $V$ magnitude from ASAS.

b Pulsational amplitude in $V$ band from ASAS.

c Szczygieł \& Fabrycky (2007).

d Values extracted from Preston et al. (2006b).

of $R \equiv \lambda / \Delta \lambda \sim 27,000$ at the $\operatorname{Mg}$ I b lines (5180 $\AA$ ). The total wavelength coverage is 3500-9000 $\AA$. Integration times ranged from a minimum value of $200 \mathrm{~s}$ (to ensure reasonably uniform illumination of the slit by starlight) to an upper limit of $600 \mathrm{~s}$ (to avoid excessive blurring of the spectrum due to changing $\mathrm{RV})$. The values of signal-to-noise ratio $(\mathrm{S} / \mathrm{N})$ achieved by such integrations can be estimated by observations of CS 22175-034 (Preston et al. 1991), which is a star with similar colors to our RR Lyr sample. Spectra of this star $(V=12.60, B-V=0.37)$ obtained near the zenith under typical observing conditions with an exposure time of $600 \mathrm{~s}$ achieved $\mathrm{S} / \mathrm{N} \sim 10$ at $4050 \AA$, $\mathrm{S} / \mathrm{N} \sim 15$ at $4300 \AA, \mathrm{S} / \mathrm{N} \sim 20$ at $5000 \AA, \mathrm{S} / \mathrm{N} \sim 30$ at $6000 \AA$, and $\mathrm{S} / \mathrm{N} \sim 30$ at $6600 \AA$. Wavelength calibrations were achieved by taking thorium-argon comparison lamp exposures at least once per hour at each star position. Basic information about our program stars is given in Table 1.

\section{DATA REDUCTION}

The raw data were bias subtracted, flat fielded, background subtracted, then extracted to one-dimensional (1D) spectra and wavelength-calibrated by use of IRAF $^{3}$ ECHELLE package. Thorium-argon identifications were based on the line list in the IRAF package data file (thar.dat) and the Th-Ar wavelength table ${ }^{4}$ provided by the LCO. We paid particular attention to scattered light corrections, and in the following subsection we describe our own (non-global) approach to this problem.

\footnotetext{
3 The Image Reduction and Analysis Facility, a general purpose software package for astronomical data, written and supported by the IRAF programming group of the National Optical Astronomy Observatories (NOAO) in Tucson, AZ.

4 http://www.lco.cl/telescopes-information/irenee-du-pont/instruments/ website/echelle-spectrograph-manuals/echelle-spectrograph-manuals/atlas
}

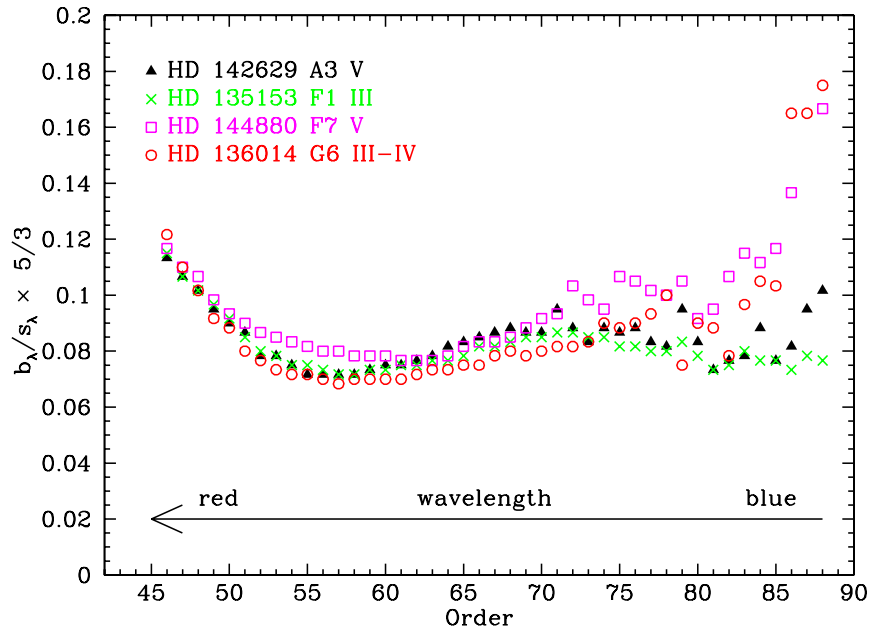

Figure 1. Fractional contribution of the inter-order background light to the on-order starlight as a function of spectral order (wavelength), $b_{\lambda} / s_{\lambda}$, for each standard star. A correction factor is applied to the $b_{\lambda} / s_{\lambda}$ ratio (see Section 3.1 for details). Wavelength decreases with increasing order.

(A color version of this figure is available in the online journal.)

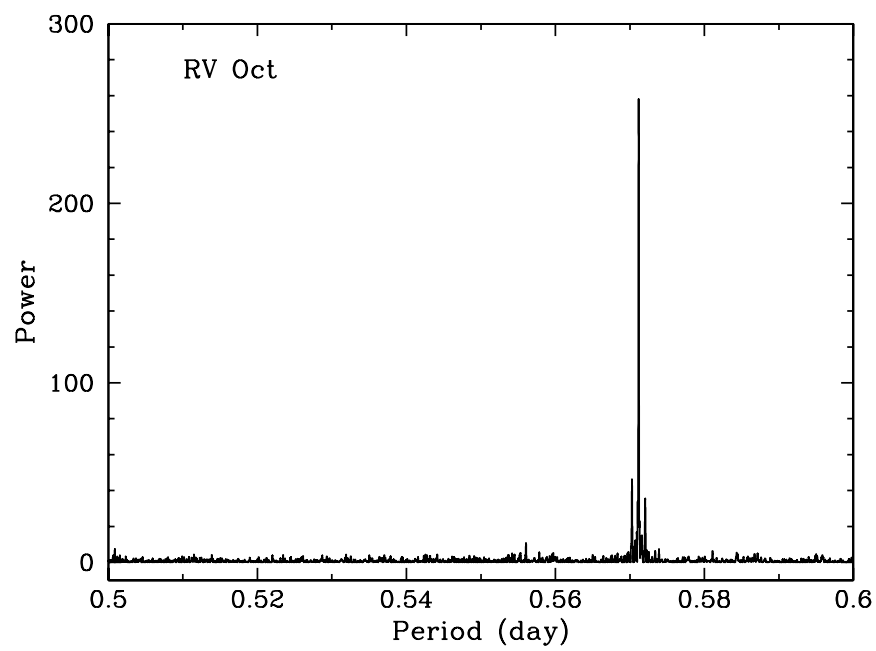

Figure 2. Example of typical periodogram used for searching the pulsational period. The sidelobes that caused by the large observing gap are clearly seen in the periodogram. The highest peak defines the pulsational period of RV Oct.

\subsection{Scattered Light Correction}

Some of the incident photons at each wavelength are scattered into all echelle orders by optical imperfections in the optical train of the spectrograph. A generic method for making scattered light corrections is use of the IRAF apscatter task, in which the scattered light pixels are fitted by a series of 1D functions across the dispersion. The independent fits are then smoothed along the dispersion by again fitting low-order functions. These fits then define the smooth scattered light surface to be subtracted from

Table 2

Basic Information and Observing Log of Standard Stars

\begin{tabular}{lcccccc}
\hline \hline Star & Spectral Type & $\begin{array}{c}\text { R.A. } \\
(\mathrm{hr} \mathrm{m} \mathrm{s})\end{array}$ & $\begin{array}{c}\text { Decl. } \\
\left({ }^{\prime \prime \prime \prime}\right)\end{array}$ & $\begin{array}{c}V \\
(\mathrm{mag})\end{array}$ & UT Date & $N_{\exp }$ \\
\hline HD 142629 & A3 V & 155653.498 & -335758.08 & 5.095 & 2008 Aug 8, 9 & 2,2 \\
HD 135153 & F1 III & 151437.319 & -313108.84 & 4.924 & 2008 Aug 9 & 3 \\
HD 144880 & F7 V & 160911.123 & -320601.20 & 7.45 & 2008 Aug 9 & 5 \\
HD 136014 & G6 III-IV & 151931.720 & -370549.78 & 6.189 & 2008 Aug 9 & 4 \\
\hline
\end{tabular}


Table 3

Mean Background Fractions $b_{\lambda} / s_{\lambda}$ for Du Pont Echelle Spectrograph

\begin{tabular}{|c|c|c|c|c|c|c|c|c|c|}
\hline Order & $\lambda_{c}{ }^{\mathrm{a}}$ & $\begin{array}{c}\text { HD } 142629 \\
\text { Mean }\end{array}$ & Mean $\times 5 / 3$ & $\begin{array}{c}\text { HD } 135153 \\
\text { Mean }\end{array}$ & Mean $\times 5 / 3$ & $\begin{array}{c}\text { HD } 144880 \\
\text { Mean }\end{array}$ & Mean $\times 5 / 3$ & $\begin{array}{c}\text { HD } 136014 \\
\text { Mean }\end{array}$ & Mean $\times 5 / 3$ \\
\hline 46 & 7575 & 0.068 & 0.113 & 0.069 & 0.115 & 0.070 & 0.117 & 0.073 & 0.122 \\
\hline 47 & 7415 & 0.064 & 0.107 & 0.064 & 0.107 & 0.066 & 0.110 & 0.066 & 0.110 \\
\hline 48 & 7260 & 0.061 & 0.102 & 0.061 & 0.102 & 0.064 & 0.107 & 0.061 & 0.102 \\
\hline 49 & 7108 & 0.057 & 0.095 & 0.058 & 0.097 & 0.059 & 0.098 & 0.055 & 0.092 \\
\hline 50 & 6962 & 0.054 & 0.090 & 0.055 & 0.092 & 0.056 & 0.093 & 0.053 & 0.088 \\
\hline 51 & 6825 & 0.052 & 0.087 & 0.051 & 0.085 & 0.054 & 0.090 & 0.048 & 0.081 \\
\hline 52 & 6688 & 0.047 & 0.078 & 0.048 & 0.080 & 0.052 & 0.087 & 0.046 & 0.077 \\
\hline 53 & 6560 & 0.047 & 0.078 & 0.047 & 0.078 & 0.051 & 0.085 & 0.044 & 0.073 \\
\hline 54 & 6435 & 0.045 & 0.075 & 0.045 & 0.075 & 0.050 & 0.083 & 0.043 & 0.072 \\
\hline 55 & 6315 & 0.043 & 0.072 & 0.045 & 0.075 & 0.049 & 0.082 & 0.043 & 0.071 \\
\hline 56 & 6202 & 0.043 & 0.072 & 0.044 & 0.073 & 0.048 & 0.080 & 0.042 & 0.070 \\
\hline 57 & 6092 & 0.043 & 0.072 & 0.043 & 0.072 & 0.048 & 0.080 & 0.041 & 0.069 \\
\hline 58 & 5987 & 0.043 & 0.072 & 0.043 & 0.072 & 0.047 & 0.078 & 0.042 & 0.069 \\
\hline 59 & 5880 & 0.044 & 0.073 & 0.044 & 0.073 & 0.047 & 0.078 & 0.042 & 0.070 \\
\hline 60 & 5780 & 0.045 & 0.075 & 0.044 & 0.073 & 0.047 & 0.078 & 0.042 & 0.071 \\
\hline 61 & 5686 & 0.045 & 0.075 & 0.045 & 0.075 & 0.046 & 0.077 & 0.042 & 0.071 \\
\hline 62 & 5592 & 0.046 & 0.077 & 0.045 & 0.075 & 0.046 & 0.077 & 0.043 & 0.071 \\
\hline 63 & 5502 & 0.047 & 0.078 & 0.046 & 0.077 & 0.046 & 0.077 & 0.044 & 0.073 \\
\hline 64 & 5413 & 0.049 & 0.082 & 0.046 & 0.077 & 0.047 & 0.078 & 0.044 & 0.073 \\
\hline 65 & 5330 & 0.050 & 0.083 & 0.047 & 0.078 & 0.049 & 0.082 & 0.045 & 0.074 \\
\hline 66 & 5250 & 0.051 & 0.085 & 0.049 & 0.082 & 0.050 & 0.083 & 0.045 & 0.076 \\
\hline 67 & 5170 & 0.052 & 0.087 & 0.049 & 0.082 & 0.050 & 0.083 & 0.047 & 0.078 \\
\hline 68 & 5090 & 0.053 & 0.088 & 0.050 & 0.083 & 0.051 & 0.085 & 0.048 & 0.080 \\
\hline 69 & 5017 & 0.052 & 0.087 & 0.051 & 0.085 & 0.053 & 0.088 & 0.047 & 0.079 \\
\hline 70 & 4945 & 0.052 & 0.087 & 0.051 & 0.085 & 0.055 & 0.092 & 0.048 & 0.080 \\
\hline 71 & 4870 & 0.057 & 0.095 & 0.052 & 0.087 & 0.056 & 0.093 & 0.049 & 0.082 \\
\hline 72 & 4805 & 0.053 & 0.088 & 0.052 & 0.087 & 0.062 & 0.103 & 0.049 & 0.082 \\
\hline 73 & 4740 & 0.050 & 0.083 & 0.051 & 0.085 & 0.059 & 0.098 & 0.050 & 0.084 \\
\hline 74 & 4672 & 0.053 & 0.088 & 0.051 & 0.085 & 0.057 & 0.095 & 0.054 & 0.090 \\
\hline 75 & 4610 & 0.052 & 0.087 & 0.049 & 0.082 & 0.064 & 0.107 & 0.053 & 0.089 \\
\hline 76 & 4548 & 0.053 & 0.088 & 0.049 & 0.082 & 0.063 & 0.105 & 0.054 & 0.090 \\
\hline 77 & 4490 & 0.050 & 0.083 & 0.048 & 0.080 & 0.061 & 0.102 & 0.056 & 0.093 \\
\hline 78 & 4430 & 0.049 & 0.082 & 0.048 & 0.080 & 0.060 & 0.100 & 0.060 & 0.100 \\
\hline 79 & 4375 & 0.057 & 0.095 & 0.050 & 0.083 & 0.063 & 0.105 & 0.045 & 0.074 \\
\hline 80 & 4320 & 0.050 & 0.083 & 0.047 & 0.078 & 0.055 & 0.092 & 0.054 & 0.090 \\
\hline 81 & 4265 & 0.044 & 0.073 & 0.044 & 0.073 & 0.057 & 0.095 & 0.053 & 0.088 \\
\hline 82 & 4210 & 0.046 & 0.077 & 0.045 & 0.075 & 0.064 & 0.107 & 0.047 & 0.078 \\
\hline 83 & 4160 & 0.047 & 0.078 & 0.048 & 0.080 & 0.069 & 0.115 & 0.058 & 0.097 \\
\hline 84 & 4110 & 0.053 & 0.088 & 0.046 & 0.077 & 0.067 & 0.112 & 0.063 & 0.105 \\
\hline 85 & 4060 & 0.046 & 0.077 & 0.046 & 0.077 & 0.070 & 0.117 & 0.062 & 0.104 \\
\hline 86 & 4012 & 0.049 & 0.082 & 0.044 & 0.073 & 0.082 & 0.137 & 0.099 & 0.166 \\
\hline 87 & 3966 & 0.057 & 0.095 & 0.047 & 0.078 & 0.123 & 0.205 & 0.099 & 0.165 \\
\hline 88 & 3920 & 0.061 & 0.102 & 0.046 & 0.077 & 0.100 & 0.167 & 0.105 & 0.175 \\
\hline
\end{tabular}

Note. ${ }^{\text {a }}$ Central wavelength of the order.

the image. Application of this method to du Pont echelle spectra is complicated by a number of considerations discussed below.

A fraction of the photons of every wavelength that passed through the $1 . .5 \times 4^{\prime \prime} .0$ entrance slit were scattered into the image plane of the du Pont spectrograph by imperfect transmission/ reflection at surfaces in the optical train. Longward of $6500 \AA$ the inter-order space became too small to measure pure scattered light. To circumvent this difficulty, we obtained observations of four standard stars through a small $0.75 \times 0.75$ slit, for which the inter-order space was more than adequate. Additional difficulties in data reduction arose due to our adopted observing procedure. Long experience at the du Pont had shown that accurate sky subtraction could not be achieved by use of light adjacent to the star image because of centering and guiding errors. If sky background is important, it must be measured
Table 4

Radial Velocities

\begin{tabular}{lcccc}
\hline \hline Star & $\begin{array}{c}\text { HJD at Midpoint } \\
(2450000+)\end{array}$ & $\begin{array}{c}\text { Phase } \\
(\phi)\end{array}$ & $\begin{array}{c}\text { RV } \\
\left(\mathrm{km} \mathrm{s}^{-1}\right)\end{array}$ & $\begin{array}{c}\text { Err } \\
\left(\mathrm{km} \mathrm{s}^{-1}\right)\end{array}$ \\
\hline CD Vel & 3836.48565 & 0.00 & 210.76 & 0.50 \\
& 3836.49453 & 0.02 & 210.97 & 0.48 \\
& 3836.54295 & 0.10 & 216.80 & 0.35 \\
& 3836.54928 & 0.11 & 218.06 & 0.35 \\
& $\ldots$ & $\ldots$ & $\ldots$ & $\ldots$ \\
& $\ldots$ & $\ldots$ & $\ldots$ & $\ldots$ \\
& $\ldots$ & $\ldots$ & $\ldots$ & $\ldots$ \\
\hline
\end{tabular}

(This table is available in its entirety in a machine-readable form in the online journal. A portion is shown here for guidance regarding its form and content.) 
Table 5

Ephemerides of Our Program Stars

\begin{tabular}{|c|c|c|c|c|c|c|}
\hline Star & $\begin{array}{c}\text { Data Used } \\
\text { (HJD 2450000+) }\end{array}$ & $\begin{array}{c}T_{0}{ }^{\mathrm{a}} \\
(\mathrm{HJD} 2450000+)\end{array}$ & $\begin{array}{c}\text { Err } \\
(\text { HJD 2450000+) }\end{array}$ & $\begin{array}{l}\text { Period }^{\mathrm{b}} \\
\text { (days) }\end{array}$ & $\begin{array}{l}\text { Period } \\
\text { (days) }\end{array}$ & $\begin{array}{l}\text { Error } \\
\text { (days) }\end{array}$ \\
\hline CD Vel & All & 3837.632 & 0.0003 & 0.57351 & 0.573510 & 0.000003 \\
\hline WY Ant & All & 4191.685 & 0.0097 & 0.57434 & 0.574344 & 0.000002 \\
\hline DT Hya & All & 4583.637 & 0.0089 & 0.56797 & 0.567978 & 0.000001 \\
\hline AS Vir & All & 4907.709 & 0.0098 & 0.553439 & 0.553412 & 0.000002 \\
\hline RV Oct & All & 3841.602 & 0.0016 & 0.571184 & 0.571170 & 0.000002 \\
\hline XZ Aps & All & 3842.735 & 0.0052 & 0.5873 & 0.587264 & 0.000002 \\
\hline BS Aps & All & 4583.785 & 0.0045 & 0.582577 & 0.582561 & 0.000007 \\
\hline \multirow[t]{2}{*}{ UV Oct } & $3836.843842 .91,4306.465021 .84$ & 3837.875 & 0.0072 & 0.542561 & 0.542578 & 0.000003 \\
\hline & $3931.584194 .92,5070.485073 .59$ & 5070.605 & 0.0072 & $\ldots$ & $\ldots$ & $\ldots$ \\
\hline \multirow[t]{3}{*}{ V1645 Sgr } & 4191.894306 .90 & 4306.775 & 0.0150 & 0.552979 & 0.552948 & 0.000005 \\
\hline & 4579.854583 .91 & 4579.895 & 0.0150 & $\ldots$ & $\ldots$ & $\ldots$ \\
\hline & $3932.733946 .75,4687.665074 .71$ & 4687.703 & 0.0170 & $\ldots$ & $\ldots$ & $\ldots$ \\
\hline Z Mic & All & 5075.606 & 0.0015 & 0.58693 & 0.586926 & 0.000001 \\
\hline TY Gru & 3945.634306 .89 & 4304.885 & 0.0120 & $\ldots$ & $\ldots$ & $\ldots$ \\
\hline
\end{tabular}

Notes.

${ }^{a}$ Epoch at time of light maxima or radial velocity minima.

${ }^{\mathrm{b}}$ Listed in ASAS catalog.

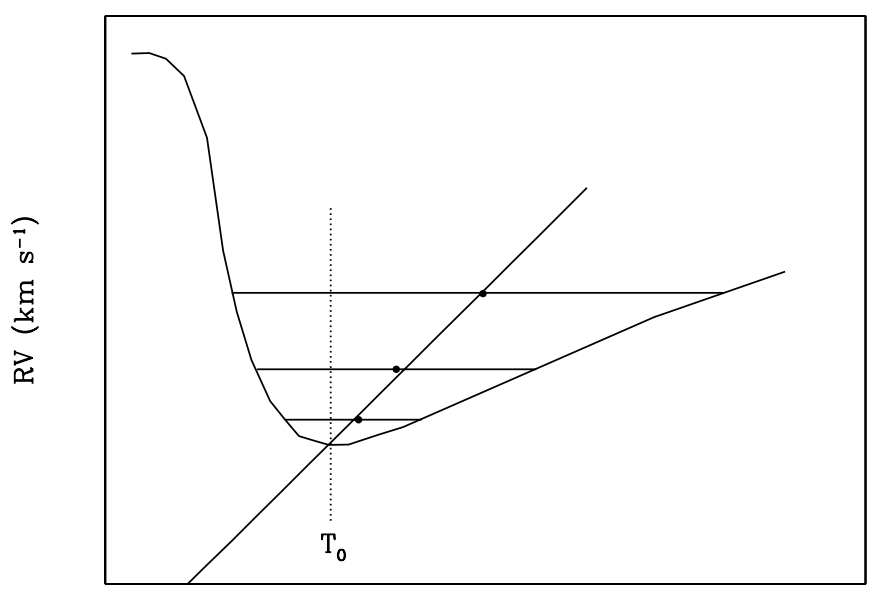

HJD

Figure 3. Schematic diagram that shows the typical radial velocity curve near minima (or equivalent to light maxima) of an RRab variable star. It also shows the Kwee-van Woerden method (Kwee \& van Woerden 1956) that we applied to determine the epochs of our RR Lyrae stars.

by sky observations before and/or after the stellar observation, and only under good photometric conditions. For stars brighter than magnitude 13 , sky was unimportant at the $1 \%$ level except near the full moon, which we avoided, so we ignored it. To save the precious time between observations that would be required to rotate the spectrograph, we made all observations with an east-west oriented slit. Furthermore, we guided with a red-sensitive detector, so that at many telescope positions significant fractions of blue-violet light did not pass through the slit due to atmospheric dispersion: the observed spectra were thus somewhat "reddened" and mimicked those of lower color temperature. In addition, this could affect the velocity difference between the red and blue lines.

To investigate such effects on our spectral line widths, we calculated the velocity shifts between spectral regions at 4000-6000 A using the following steps: (1) calculate the parallactic angle for each of our stars at different $7 \mathrm{hr}$ angles (from 0.01 to $6 \mathrm{hr}$ with increment of $1 \mathrm{hr}$ ); (2) calculate the
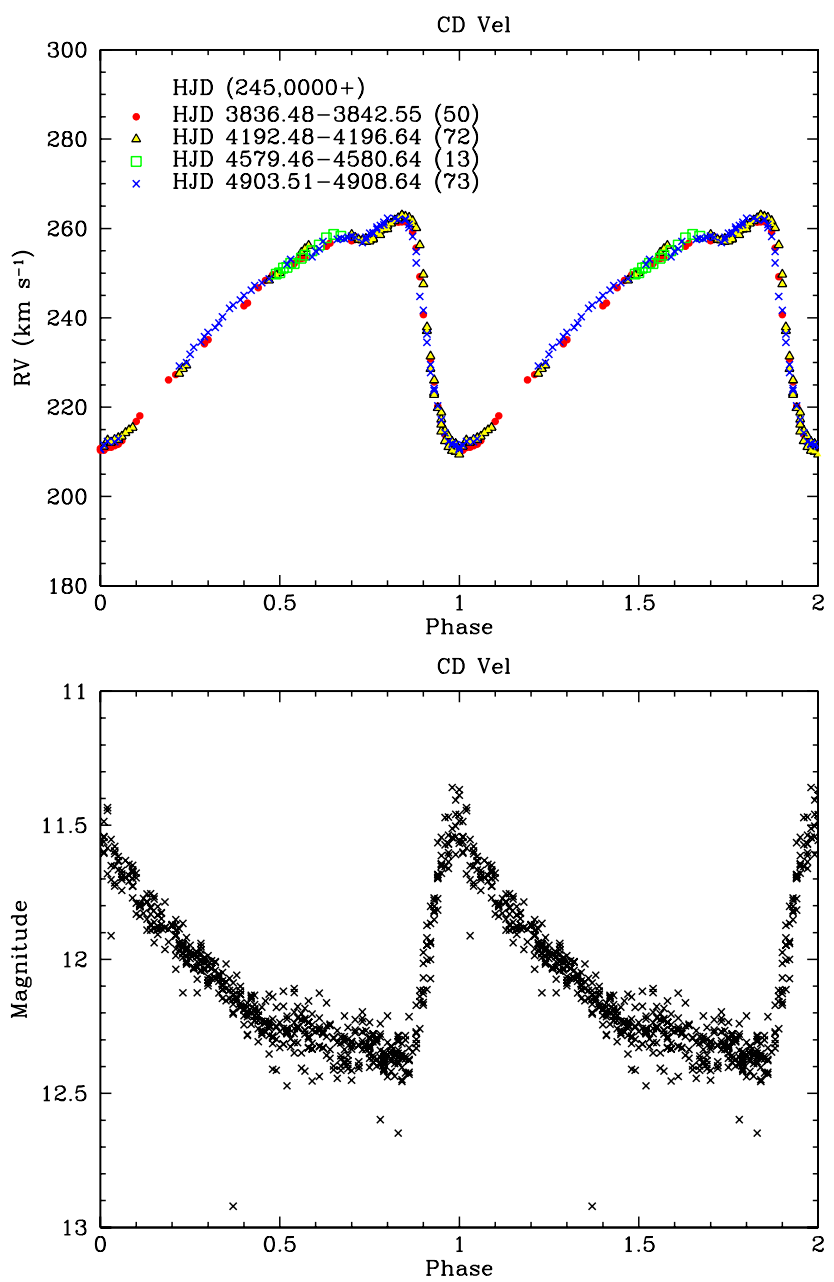

Figure 4. Folded radial velocity curve and light curve of CD Vel by using our derived ephemeris for this star (Table 5). Top panel: radial velocity vs. pulsational phase for all of our spectra. The different symbols and colors represent different times of observations in HJD. The total numbers of observed spectra per cycle are listed in the parentheses. Bottom panel: the ASAS photometric light curve vs. pulsational phase. The scatter of data points at a given phase for $\mathrm{CD} \mathrm{Vel}$ and for the other program stars is highly related to the mean apparent brightness of the observed star.

(A color version of this figure is available in the online journal.) 

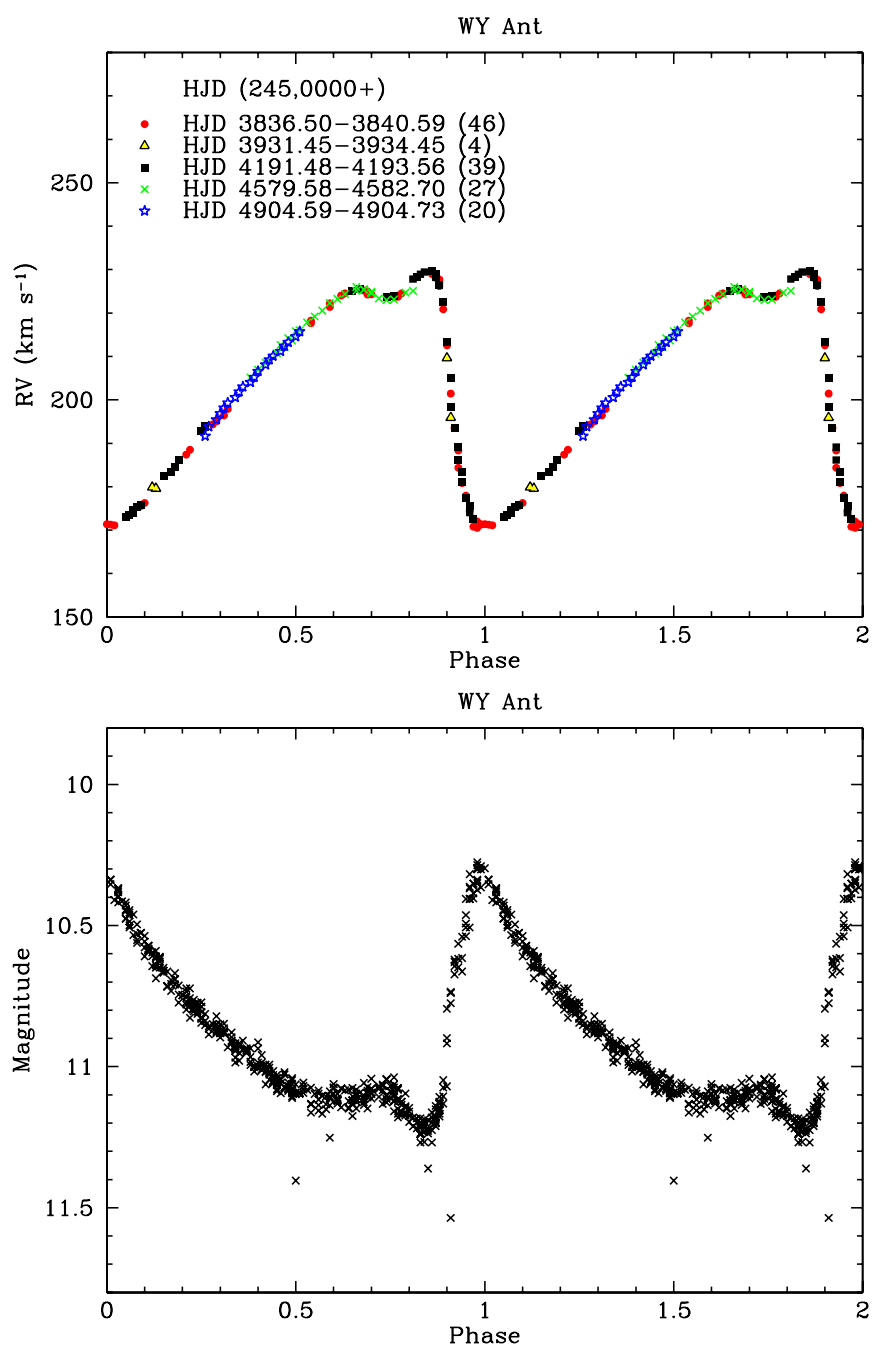

Figure 5. Same as Figure 4.

(A color version of this figure is available in the online journal.)

angle between the east-west slit of our Cassegrain spectrograph on equatorially mounted telescope, and direction to zenith; (3) calculate the sine of the inclination of the spectrum to the slit; (4) calculate the altitude of each star; (5) calculate differential atmospheric dispersion by linear approximate of data shown in Figure 2 of Simon (1966) for elevation 2811 m, which is close to du Pont elevation at $2200 \mathrm{~m}$; (6) calculate the differential atmospheric dispersion perpendicular to the slit; (7) finally, convert angular displacement in arcseconds to velocity displacement by use of scale factor $8 \mathrm{~km} \mathrm{~s}^{-1} \operatorname{arcsec}^{-1}$ (assuming $1^{\prime \prime} .5$ slit width projects to $12 \mathrm{~km} \mathrm{~s}^{-1}$ ). The upper limit of $6 \mathrm{~km} \mathrm{~s}^{-1}$ was set for the conversion, which corresponds to an illumination centroid at the edge of the slit. During the observation, seeing and guiding errors will diminish atmospheric displacements, e.g., producing centroids nearer to aperture center. Our velocity displacement calculations over $7 \mathrm{hr}$ angles of each star range from 0 to $2 \mathrm{~km} \mathrm{~s}^{-1}$, which are small compared with intrinsic RR Lyr line width of $>20 \mathrm{~km} \mathrm{~s}^{-1}$. Thus, the broadening effects of these displacements are small on individual spectra.

To further investigate whether such broadening would have any effect on the co-added spectrum, we measured the equivalent widths of several metal lines of individual spectrum and co-added spectrum of the same phase. The comparisons of measured equivalent width are consistent with an overall difference
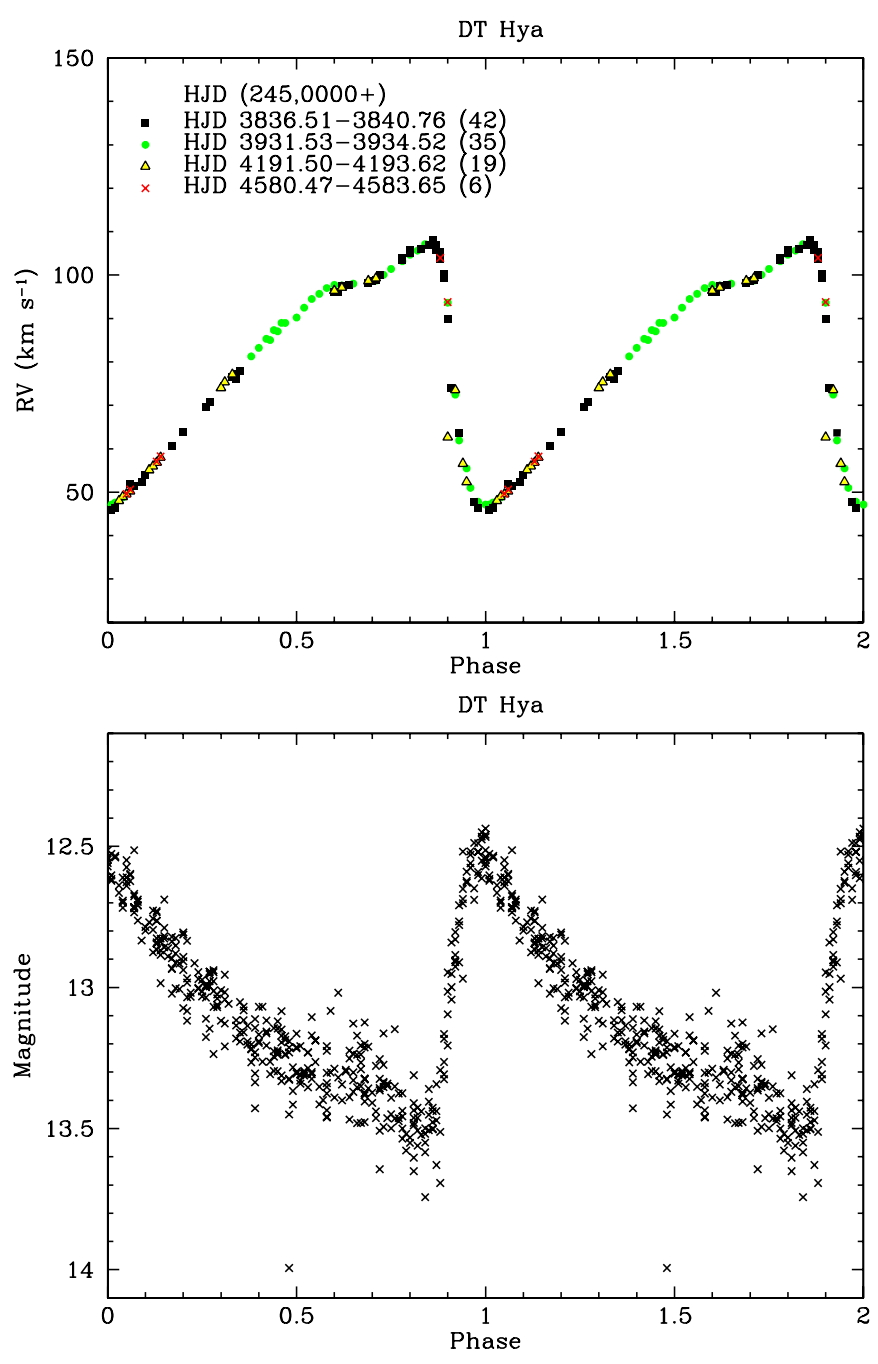

Figure 6. Same as Figure 4.

(A color version of this figure is available in the online journal.)

of $\pm 3 \mathrm{~m} \AA$. As such, we conclude that the equivalent widths are unaffected in these cases. However, we warn the reader that the shifts certainly contribute to systematic errors of individual RVs, especially for stars with large Southern declinations (decl. < -70). Inspecting the scatter of RV data for the stable RRab stars (WY Ant, DT Hya, CD Vel, XZ Aps, RV Oct, and $\mathrm{Z}$ Mic) of our RV curves, the errors due to blue image decentering cannot be much greater than $1 \mathrm{~km} \mathrm{~s}^{-1}$.

The raw spectra of the observed standard stars were bias subtracted and flat fielded. Then, individual spectra were combined into a single spectrum. We extracted each combined spectrum with a 6 pixel aperture to two 1D spectra, with one for the star and one for inter-order background. Subsequently, the 1D spectra were continuum normalized with the continuum task in IRAF ECHELLE package. To obtain the contribution of scattered light in each order, we calculated the fractional contribution of the inter-order background light to the on-order starlight as a function of spectral order (or wavelength), $b_{\lambda} / s_{\lambda}$, for each standard star. Because a 10 pixel aperture was used to extract the spectra of our program RR Lyr, the extracted 1D spectra are expected to contain more scattered light than the extracted scattered light frames. Thus, we applied a correction factor of $5 / 3$ to the calculated $b_{\lambda} / s_{\lambda}$ ratios. 

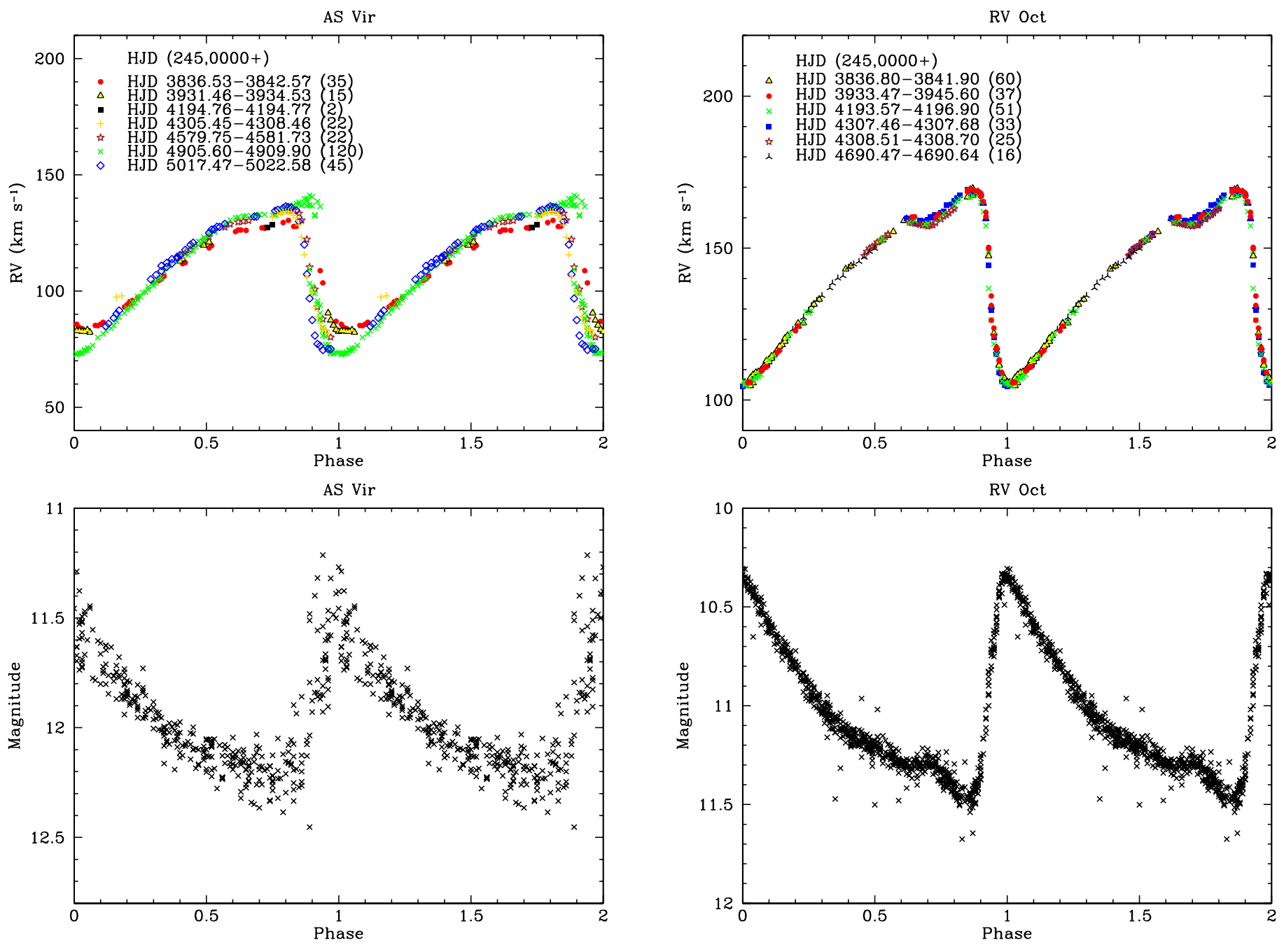

Figure 7. Same as Figure 4.

(A color version of this figure is available in the online journal.)

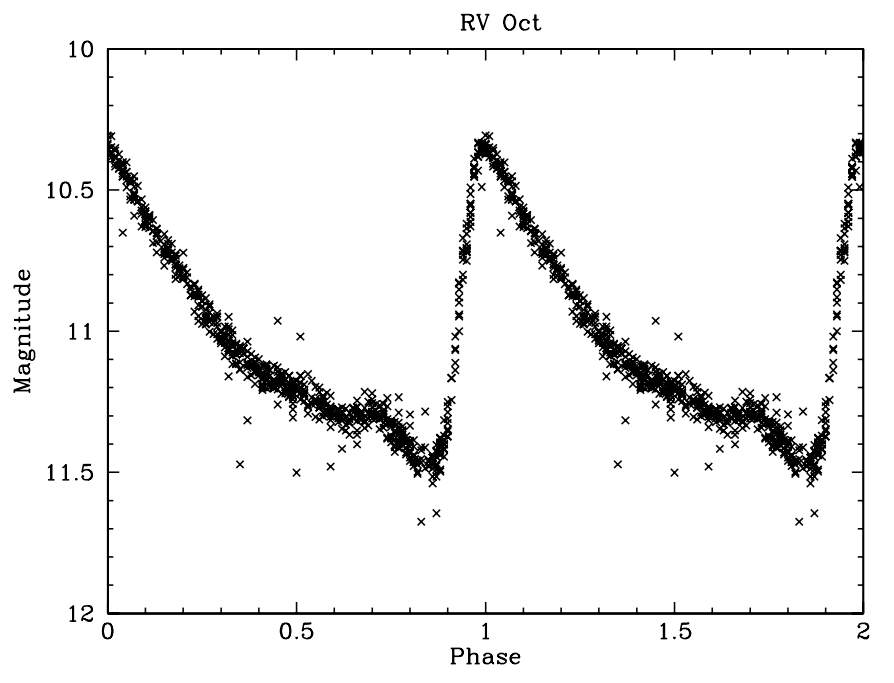

Figure 8. Same as Figure 4.

(A color version of this figure is available in the online journal.)

In Table 2, we provide the basic information and observing $\log$ for our standard stars. The calculated fraction as a function of spectral order for each standard star is presented in Table 3. We summarize our results in Figure 1. The success of this calibration procedure depends on the stability of the scattered light distribution produced by the spectrograph. Recalibration performed from time to time by the procedure described above has shown that the scattered light distribution has changed little, if at all, during the past two decades. We will consider this issue and describe the subtraction of scattered light from the spectra of RR Lyr in a subsequent paper (B.-Q. For et al. 2011, in preparation).

\section{ANALYSIS}

\subsection{Radial Velocities}

The spectra that we used for deriving the RVs were not corrected for scattered light. It is not important for deriving the RVs but will be required for the subsequent atmospheric analysis. We derived the RVs by use of the cross-correlation FXCOR task in IRAF, in which the individual spectra were cross-correlated against a template by fitting a Gaussian to the cross-correlation peak. We constructed the individual spectra from 13 echelle orders covering the spectral region of $4000-4600 \AA$, which were then flattened, normalized, and stitched together with an IRAF script. In order to get strong cross-correlations that minimize $\mathrm{RV}$ errors, we created a template from several spectra of CS 22874-009, a blue metal-poor RV standard star (Preston \& Sneden 2000), which possesses a spectrum similar to those of RR Lyr stars at most phases. The typical RV error calculated from FXCOR is $\sim 0.5 \mathrm{~km} \mathrm{~s}^{-1}$. We present the observed HJD midpoints, phases (see Section 4.2), derived RVs, and their associated errors in Table 4.

\subsection{Pulsation Ephemerides}

A pulsation ephemeris is commonly written as HJD (max light) $=T_{0}+n \times \mathrm{P}$, where $T_{0}$ is epoch, $n$ is the number of elapsed pulsation cycles, and $P$ is the pulsational period in days. The All Sky Automated Survey ${ }^{5}$ (ASAS; Pojmanski 2002) provides a starting point to obtain ephemerides for our program stars. This photometric survey has been carried out over many years at the LCO and Haleakala, Maui stations. Using the ASAS reported pulsation period and $T_{0}$, the folded light curves as shown on the ASAS Web site were slightly out of phase. This suggests that the quoted values could be improved. Here, we present the

\footnotetext{
5 http://www.astrouw.edu.pl/asas/
} 

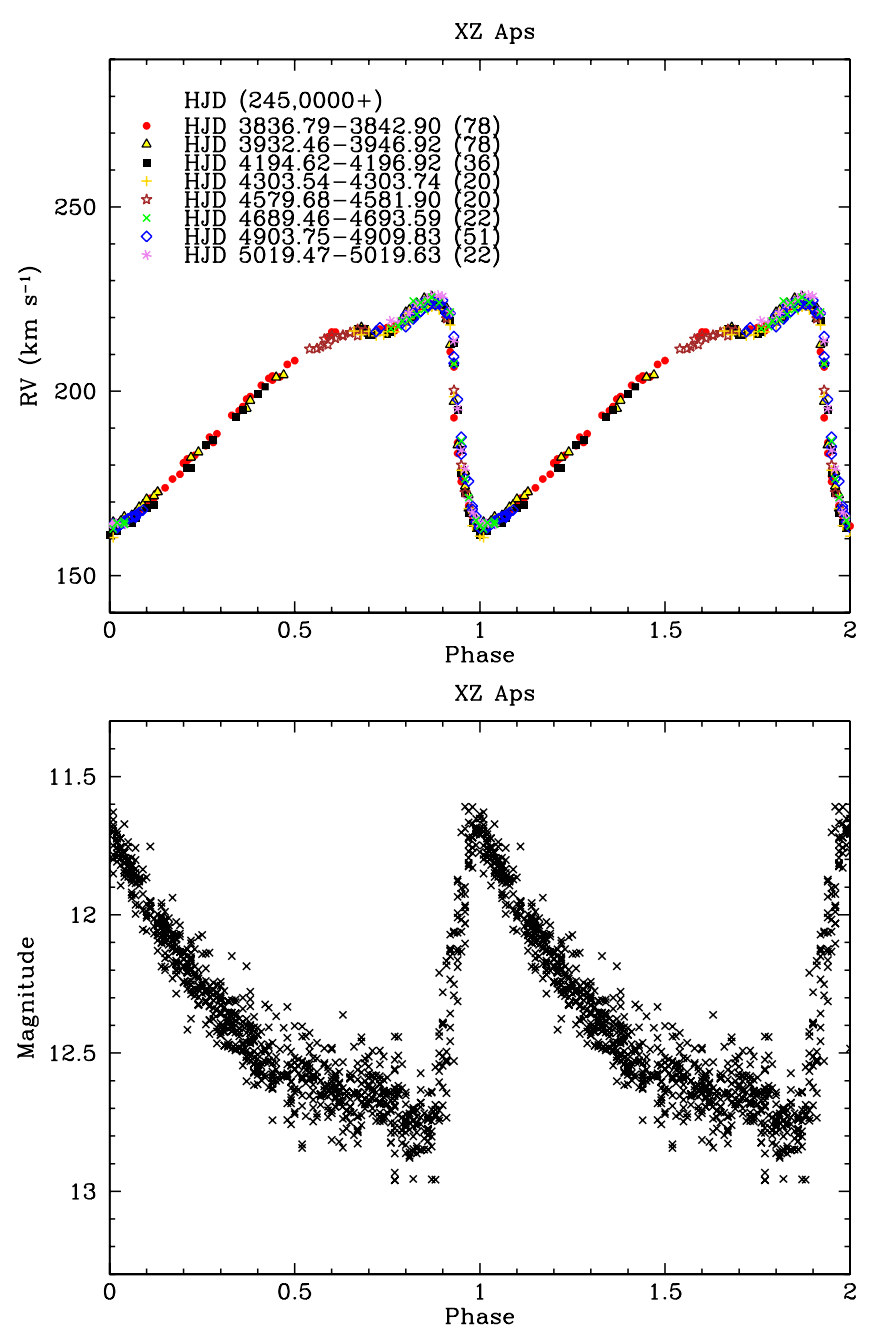

Figure 9. Same as Figure 4.

(A color version of this figure is available in the online journal.)

methods of improving both pulsation periods and $T_{0}$ values of our program stars.

\subsubsection{Pulsation Period}

We improved the pulsation periods of our 10 RRab stars using the classified "grade A" $V$-band photometric data listed in the ASAS database. The pulsation period of TY Gru was adopted from Preston et al. (2006b) since those authors derived it by use of additional observations obtained with the LCO Swope telescope. The pulsational periods were derived using the Lomb-Scargle periodogram (Scargle 1982). We set a short period range of $0.5-0.6$ days to minimize the chance of selecting spurious peaks caused by aliasing sidelobes (due to large observational gap and unevenly spaced time series data) in a different frequency domain. In addition, the pulsational period of our RRab stars is known within this range, in which a smaller time step can be set to achieve accuracy while cutting down the computing time. The advantages of this algorithm are: (1) less computing time than the light curve template fitting method, which requires continuous sampled data sets that are not available from the ASAS database and (2) the ability to compute Fourier transforms for unevenly spaced time series data. While we have continuous sampled RV data, we still cannot use the template fitting method because it is designed for light curves fitting not for RV curves of RR Lyr stars. We warn the reader
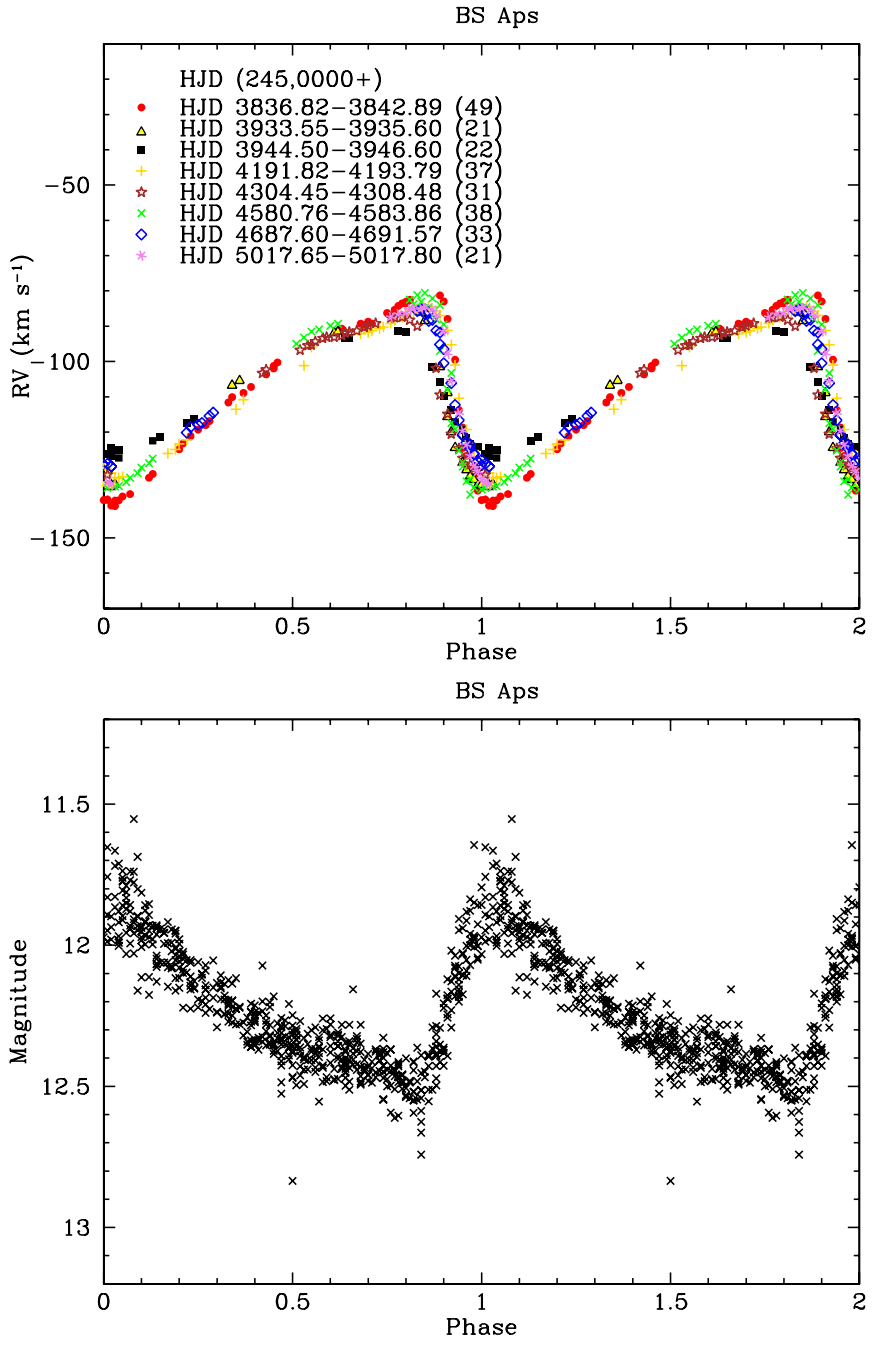

Figure 10. Same as Figure 4.

(A color version of this figure is available in the online journal.)

that there is a caveat for this algorithm. It is optimized to identify sinusoidal-shaped periodic signal in time-series data. The light curves of RR Lyrae stars are non-sinusoidal.

Given that we have huge amount of photometric data, the highest peak, which represents the most probable repeating signal, in a periodogram is always more than $4 \sigma$ above the mean noise level (see Figure 2). The highest peak of each periodogram is selected as the pulsational period of our program stars. We evaluated the error of the periods by comparing the periods derived from Lomb-Scargle algorithm and box-fitting leastsquares method (BLS; Kovács et al. 2002). The BLS algorithm fits the input time series with "box"-shaped function, which makes it more suitable for obtaining period for transiting light curve than RR Lyr light curve.

In Table 5, we present the pulsation periods quoted in the ASAS catalog in Column 5, the derived pulsation periods and their associated errors in Columns 6 and 7. The error of the period is within $0.000001-0.000007$ days, which is 10 times better than the periods accuracy quoted at the ASAS Web site.

\subsubsection{Epoch}

The reference epoch $\left(T_{0}\right)$ of a pulsating variable star is usually chosen to occur at visual light maximum, which closely coincides with RV minimum (see discussion by Preston 2009 

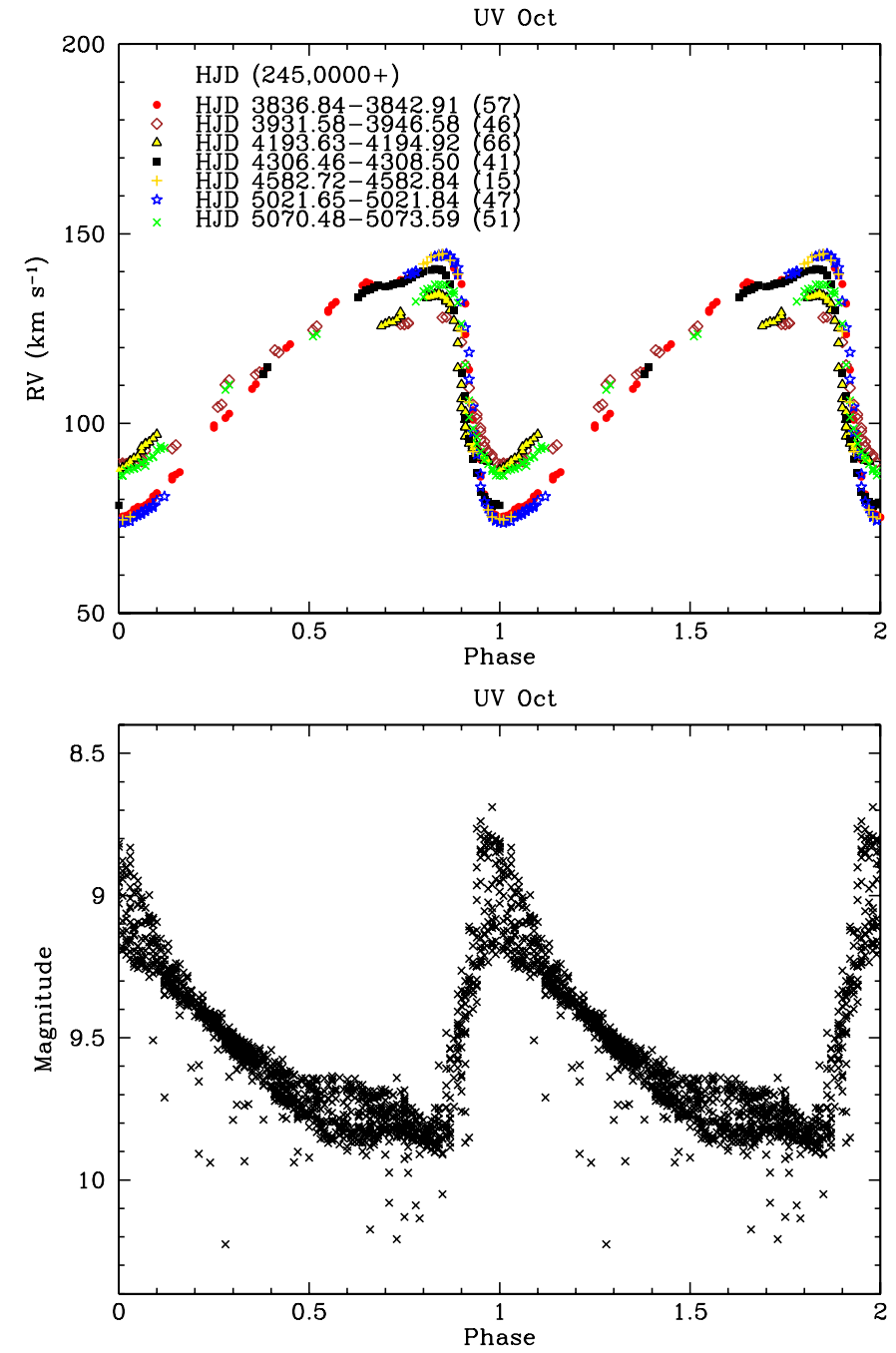

Figure 11. Same as Figure 4.

(A color version of this figure is available in the online journal.)

and particularly Figures 4-14). Because the periodogram does not calculate an epoch, we derived values of $T_{0}$ by use of the Kwee-van Woerden method (Kwee \& van Woerden 1956). This method is generally used for computing the epoch of minimum of eclipsing variables accurately but it is also suitable to determine the epoch of light maxima of variable stars. We prefer to use our RV curves for this purpose because adequate data points near the RV minima (light maxima) were available during individual cycles, in contrast to the ASAS light curve data that were collected over long time intervals, with few observations per cycle and relatively large scatter near light maxima.

For each star, we selected the cycles that cover the RV minima and calculated several equidistant midpoints between the rising and descending branch near the RV minima for a given cycle. Then, we fitted a linear least-squares equation to these midpoints, which the intersection of the straight line and the RV curve gives the $T_{0}$ of RV minima. We typically computed more than one $T_{0}$ per star using the above method to evaluate the error. Assuming the pulsational period and the first derived $T_{0}$ are accurate, we can calculate the predicted $T_{0}$ after $n$ pulsation cycles using the defined pulsation ephemeris above.
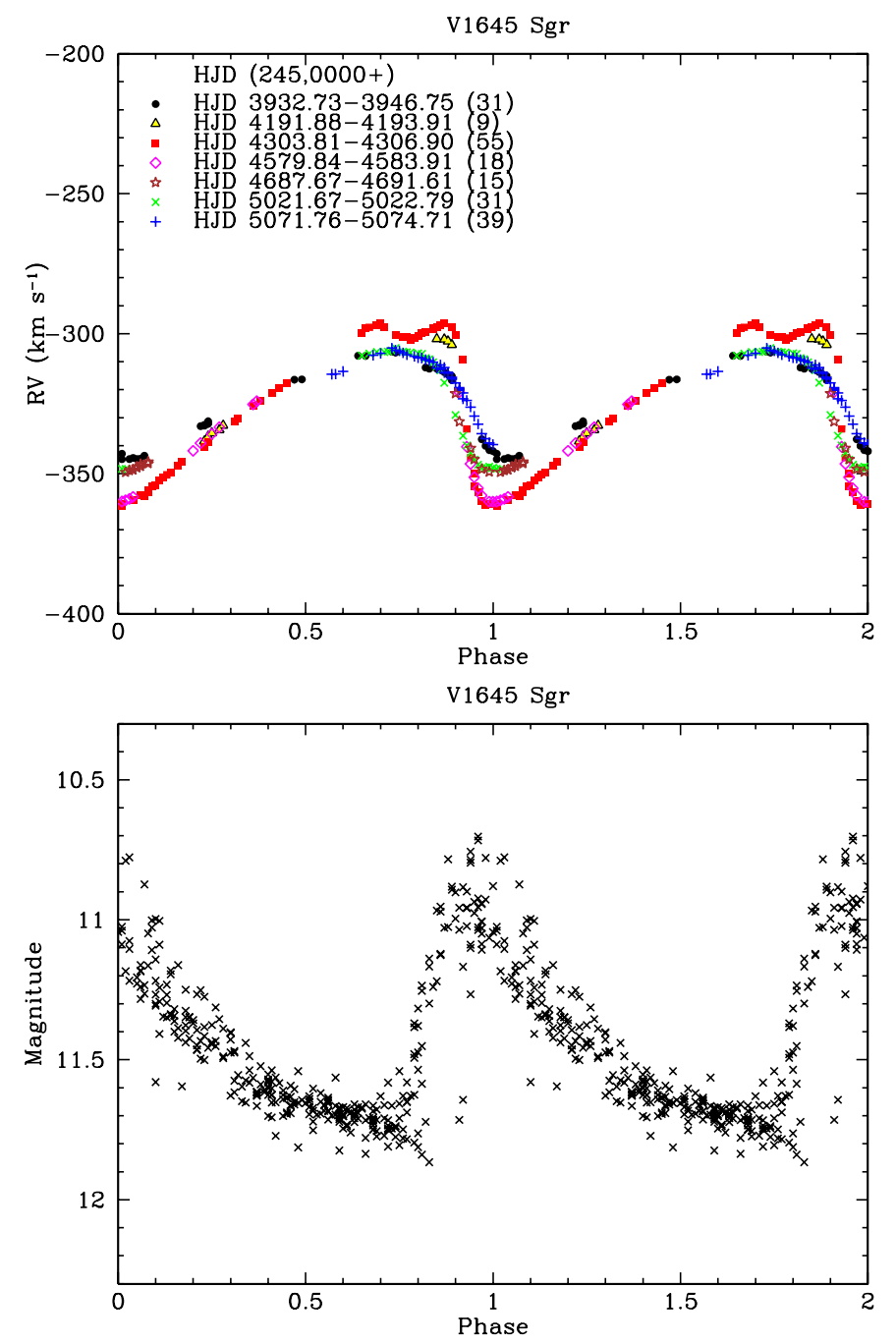

Figure 12. Same as Figure 4.

(A color version of this figure is available in the online journal.)

The predicted $T_{0}$ should be close to the second derived $T_{0}$. The difference between the predicted and the derived value provides an estimate for the error. Due to the possibility of period change for the Blazhko RRab stars, several epochs were determined and used for folding their RV curves.

In Figure 3, we show the schematic diagram that determines the times of RV minima of our asymmetric RV curves. We refer the reader to Kwee \& van Woerden (1956) for the mathematical description of the method (for symmetric light curve only). In Table 5, we summarize the ephemerides of 11 field RRab stars. We tabulate epochs for the particular RV minima used to derive them. The table also gives the range of data in HJD that are associated with the corresponding $T_{0}$ and pulsational periods. We present the folded RV curves and ASAS light curves with our derived ephemerides in top and bottom panels, respectively, of Figures 4-14. The figures are arranged by ascending right ascension.

B.-Q.F. acknowledges travel assistance from a SigmaXi grant-in-aid. This research was supported by U.S. National Science Foundation grant AST-0908978. 

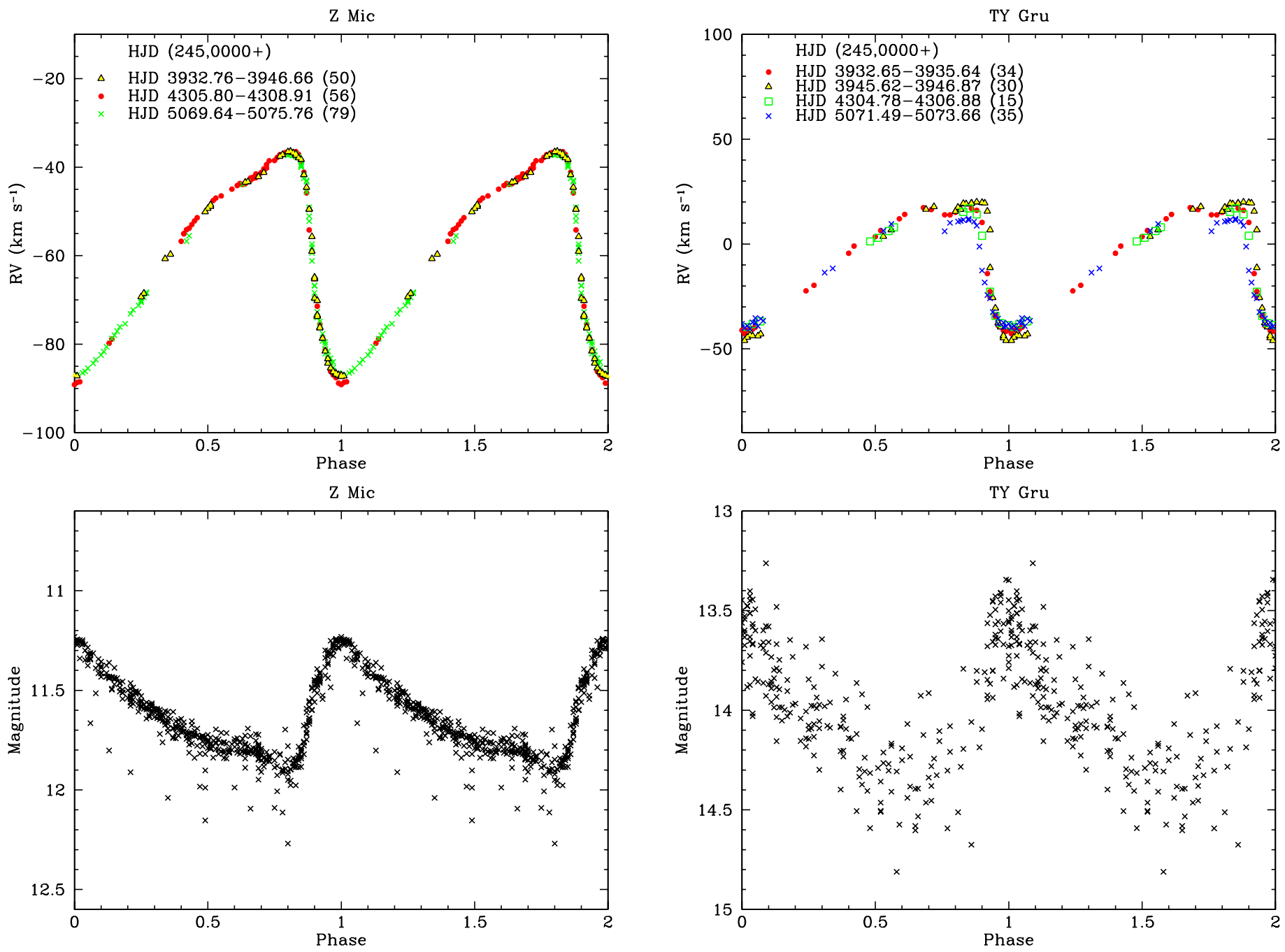

Figure 13. Same as Figure 4.

(A color version of this figure is available in the online journal.)

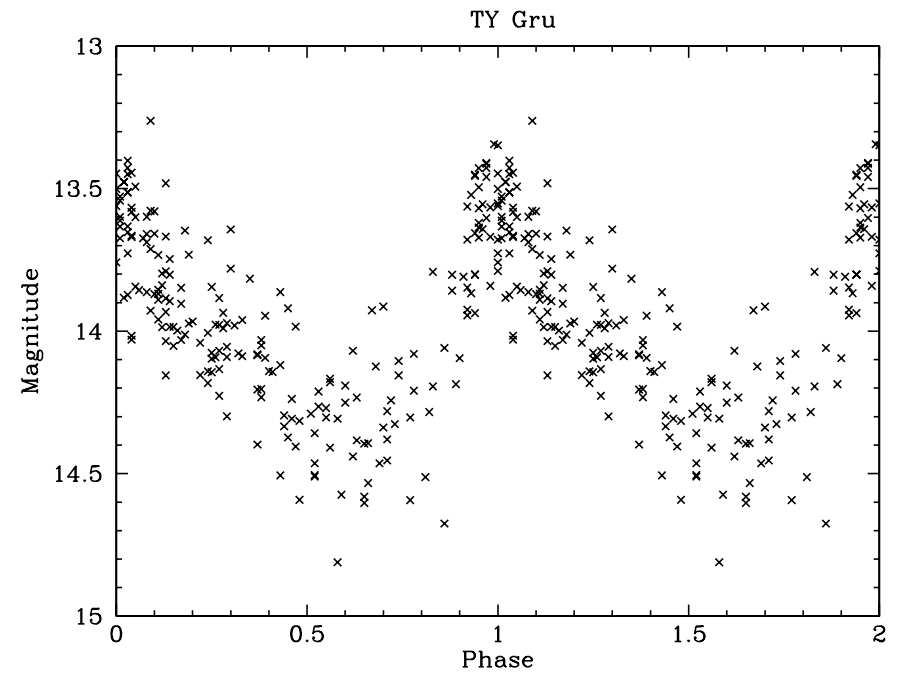

Figure 14. Same as Figure 4.

(A color version of this figure is available in the online journal.)

\section{REFERENCES}

Beers, T. C., Preston, G. W., \& Shectman, S. A. 1992, AJ, 103, 1987

For, B.-Q., \& Sneden, C. 2010, AJ, 140, 1694

Ivezić, Ž., et al. 2004, in ASP Conf. Ser. 327, Satellites and Tidal Streams, ed.

F. Prada, D. Martinez Delgado, \& T. J. Mahoney (San Francisco, CA: ASP), 104

Kovács, G., Zucker, S., \& Mazeh, T. 2002, A\&A, 391, 369

Kwee, K. K., \& van Woerden, H. 1956, Bull. Astron. Inst. Netherlands, 12, 327

Majewski, S. R., Skrutskie, M. F., Weinberg, M. D., \& Ostheimer, J. C. 2003, ApJ, 599, 1082

Pojmanski, G. 2002, Acta Astron., 52, 397

Preston, G. W. 1959, ApJ, 130, 507

Preston, G. W. 2009, A\&A, 507, 1621

Preston, G. W. 2011, AJ, 141, 6

Preston, G. W., Shectman, S. A., \& Beers, T. C. 1991, ApJS, 76, 1001

Preston, G. W., \& Sneden, C. 2000, AJ, 120, 1014

Preston, G. W., Sneden, C., Thompson, I. B., Shectman, S. A., \& Burley, G. S. 2006a, AJ, 132, 85

Preston, G. W., Thompson, I. B., Sneden, C., Stachowski, G., \& Shectman, S. A. 2006b, AJ, 132, 1714

Scargle, J. D. 1982, ApJ, 263, 835

Simon, G. W. 1966, AJ, 71, 190

Szczygieł, D. M., \& Fabrycky, D. C. 2007, MNRAS, 377, 1263

Vivas, A. K., et al. 2004, AJ, 127, 1158 